\title{
Cuneate Nucleus
}

National Cancer Institute

\section{Source}

National Cancer Institute. Cuneate Nucleus. NCI Thesaurus. Code C52716.

One of the dorsal column nuclei in the medulla oblongata, which is part of posterior column-medial lemniscus pathway, carrying sensory information from most the upper body to the contralateral thalamus via the medial lemniscus. 